\title{
Presentation, Evaluation, Management, and Outcomes of Acute Stroke in Low- and Middle-Income Countries: A Systematic Review and Meta-Analysis
}

\author{
Rasha Khatib ${ }^{a}$ Yurany A. Arevalo ${ }^{a}$ Mark A. Berendsen ${ }^{b}$ Shyam Prabhakaran ${ }^{a}$ \\ Mark D. Huffman ${ }^{c}$ \\ a Department of Neurology, Northwestern University Feinberg School of Medicine, Chicago, IL, USA; \\ ${ }^{b}$ Galter Health Sciences Library, Northwestern University Feinberg School of Medicine, Chicago, IL, USA; \\ 'Department of Preventive Medicine, Northwestern University Feinberg School of Medicine, Chicago, IL, USA
}

\section{Keywords}

Stroke - Low- and middle-income countries .

Acute care $\cdot$ In-hospital mortality $\cdot$ Evidence-based

practice $\cdot$ Meta-analysis

\begin{abstract}
Aims: We performed a systematic review to evaluate stroke presentation, evaluation, management, and outcomes among studies conducted in low- and middle-income countries (LMIC). Methods: We searched MEDLINE (Ovid), Embase (Elsevier), and the Global Health (EBSCOhost) databases between January 2005 and June 2017 for studies conducted in LMICs defined by the World Bank. We pooled prevalence estimates using an inverse-variance weighting method and stratified by the country income group. Results: The search identified 36 hospital-based studies (64,256 participants) in LMICs. Mean (SD) age ranged from 51 (14) to 72 (12) years, and $29-56 \%$ of patients were women. Hypertension was the most commonly reported risk factor (64\% [95\% Cl 59-69]). In settings where MRI was not used, head CT scans were reported among $90 \%$ patients (95\% Cl 79-97). Overall, 3\% $(95 \% \mathrm{Cl} 2-4)$ of patients were treated with tissue plasmino-
\end{abstract}

() 2018 S. Karger AG, Basel

\section{KARGER
E-Mail karger@karger.com
www.karger.com/ned \\ KARGER
E-Mail karger@karger.com
www.karger.com/ned}

gen activator, and $78 \%(95 \% \mathrm{Cl} 66-88)$ were treated with antiplatelet therapy. Overall, the rate of in-hospital mortality was $14 \%$ (95\% Cl 10-19), and the rate of in-hospital pneumonia was $17 \%(95 \% \mathrm{Cl} 14-20)$. Conclusions: Our review revealed the low use of evidence-based practices for acute stroke care in LMIC. The true use in hospitals that do not conduct this research is probably even lower. Strategies to evaluate and improve health system performance for acute stroke care, including implantation of stroke units and making thrombolysis more available and affordable are needed in LMIC. Registration in Prospero: CRD42017069325.

๑ 2018 S. Karger AG, Basel

\section{Background}

In 2015, there were an estimated 42 million prevalent cases of cerebrovascular disease worldwide, including an estimated 5.39 (95\% uncertainty interval [UI]: 5.02-5.73) million acute first ischemic strokes, and 3.58 (95\% UI 3.34-3.82) million acute first hemorrhagic and other strokes [1]. Estimates from the Global Burden of Diseases, Injuries, and Risk Factors Study (GBD 2016) 
ranked stroke as the second most common cause of deaths [2].

Stroke care has undergone a major transformation in the past decade with the emergence of evidence-based approaches to stroke prevention, acute stroke management, and stroke rehabilitation [3]. However, stroke systems of care and the availability of resources for acute stroke care vary considerably across geographic regions, which leads to uneven and at times, suboptimal care [4]. Evidencebased approaches for improving acute stroke care have been implemented in high-income countries (HIC) with limited implementation in low- and middle-income countries (LMIC) [4]. As a result, morbidity and mortality rates from acute stroke are higher in LMIC compared to HIC [1].

The United Nations and World Health Organization (WHO) member organizations have committed to efforts to reduce premature mortality due to non-communicable diseases by one-third by 2030 [5]. Premature morbidity and mortality from stroke could be significantly reduced through hospital-based acute stroke quality improvement programs, including the implementation of evidence-based clinical practice guidelines, [3] and dedicated stroke units [4].

Although many HIC have adopted these programs, the development, implementation, and evaluation of such programs in LMIC remain low [4]. To identify the potential gains from optimal acute stroke management, we performed a systematic review to evaluate stroke presentation, evaluation, management, and outcomes in LMIC. Our evaluation of acute stroke management is guided by the Global Stroke Services Action Plan developed by the World Stroke Organization, [6] which identifies key evidencebased practices across a continuum of healthcare models in which these practices may be realistically carried out.

\section{Methods}

\section{Search Strategy}

We performed this systematic review in accordance with the Preferred Reporting Items for Systematic Reviews and MetaAnalyses guidelines [7]. Details of the protocol for this systematic review were prospectively registered on PROSPERO (CRD42017069325). On June 7, 2017, we searched MEDLINE (Ovid), Embase (Elsevier) and the Global Health (EBSCOhost) databases. We limited retrieval to articles published between January 2005 and June 2017, since most advances in stroke have been made in the past decade, and previous publications may not provide information on what is currently evidence based.

In each database, we used subject headings and title abstract keywords descriptive of concepts related to the presentation and management of stroke in LMIC. We modified the LMIC filter developed by the Cochrane Effective Practice and Organization of Care [8] to include countries classified by the World Bank as LMIC as of March 2017 [9]. We used a study filter which incorporated and expanded upon terminology used in the cohort and cross-sectional study filters developed by the University of Texas School of Public Health [10]. We limited retrieval in MEDLINE and Embase to studies of human subjects and to "human diseases" in the Global Health database. We also manually searched reference lists of reviews and relevant publications. We did not restrict the search by language. The database search strategies are presented in the online supplementary Table I (for all online suppl. material, see www.karger.com/ doi/10.1159/000491442).

\section{Study Selection}

Two reviewers (R.K. and Y.A.A.) independently screened titles, abstracts, and full texts of retrieved articles based on pre-specified inclusion and exclusion criteria. Disagreements were resolved by consensus. Articles were included if they fulfilled the following criteria: Population: In-hospital adult populations from LMIC with acute stroke (ischemic or hemorrhagic); Outcomes: Studies that provided information on at least 2 of the following: presentation of stroke, acute management of stroke, or stroke outcomes; Study design: Observational, hospital-based cohorts, cross-sectional studies, registries, and hospital databases. Intervention studies (e.g., randomized controlled trials, before and after studies) and studies in community settings were excluded.

\section{Data Abstraction}

One reviewer (Y.A.A.) extracted data using a pretested data abstraction form, and these data were checked by another reviewer (R.K.) to assess accuracy. The data collected included study characteristics (study design, country, and data collection period), cohort characteristics (type of stroke and any cohort restrictions), stroke presentation (stroke risk factors, level of consciousness and other scales of disability at admission, and swallow function assessment), stroke evaluation and management (Imaging, Thrombolysis and antiplatelet therapy, Stroke and intensive care unit admissions, Rehabilitation and early in hospital mobilization), and outcomes (in-hospital mortality, and in-hospital pneumonia).

\section{Risk of Bias in Individual Studies}

Two authors (R.K. and Y.A.A.) independently evaluated the methodological and reporting quality of each study using a modified version of the Newcastle-Ottawa Scale that has been used in similar systematic reviews [11]. The risk of bias was assessed based on 4 study areas that are relevant to the study design of included studies: selection bias (selection of study participants), performance bias (pre-defined stroke criteria based on objective measures), detection bias (outcome assessment), and attrition bias (completeness of follow-up). Funnel plots and Eggers test were used to assess potential publication bias if at least 10 studies were available [12].

\section{Synthesis of Results}

The individual study estimates extracted were combined when estimates were available from more than 3 studies. Data were pooled using the inverse-variance weighting method to provide information on the prevalence with corresponding 95\% CIs. Similar methods of pooling estimates on presentation, management, and outcomes have been used for reviews of other diseases, including heart failure [13]. We used similar methods with the expectation that heterogeneity across studies will be high. Results are pre- 
sented as forest plots and 95\% CIs, stratified by the country income group, using World Bank definitions [9]. Heterogeneity was assessed by the $I^{2}$ statistic, and statistical significance was defined as a 2 -sided $p<0.05$. We conducted analyses using STATA version 10.1 (StataCorp, LLC, College Station, TX, USA).

\section{Results}

\section{Search Results}

We identified 6,599 non-duplicate citations from our search. Based on the title and abstract screening, we excluded 5,500 citations. We excluded an additional $138 \mathrm{ci}$ tations based on full-text screening. Reasons for excluding full text citations were conference abstracts, studies with incomplete results, wrong populations (e.g., HIC settings, acute coronary syndrome), studies with duplicate data, and reviews. After exclusion of citations that were not relevant, 36 studies were included (online suppl. Fig. I ).

\section{Study Characteristics}

We included 36 eligible studies that totaled 64,256 patients. Details of study characteristics, divided by region, are included in online supplementary Table II. Most studies were conducted in upper middle-income countries (UMIC) and lower middle-income countries (LrMIC), and only 4 (11\% of studies) were conducted in low-income countries (LIC). The sample size in the included studies ranged from 42 patients from South Africa to 31,274 patients from China. The mean (SD) age of patients ranged from 51 (14) years in India to 72 (12) years in Lebanon. Online supplementary Figure II lists the mean age in each study ranked by the Gross National Income and grouped by the country income group. The percentage of male patients ranged from $44 \%$ in China to $71 \%$ in Serbia and China. Selection criteria varied by study. Five studies included only stroke patients who received recombinant tissue plasminogen activator (rt-PA) treatment. Many studies included any type of stroke (56\%), while others restricted inclusion to ischemic strokes only (43\%); one study restricted inclusion to hemorrhagic strokes only. Online supplementary Figure III presents the pooled proportion of hemorrhagic strokes by the country income group among studies in which the inclusion criteria was not restricted by the type of stroke ( $n=18$ studies).

\section{Risk of Bias of Individual Studies}

A summary of risk of bias assessment of each study is presented in online supplementary Table III. In forty-nine percent ( $n=17$ studies) of studies, consecutive sampling was utilized to select study patients to minimize the risk of selection bias. A clear criteria to define a stroke was stated in $66 \%$ ( $n=23$ studies) of included studies. Most commonly cited criteria included the WHO criteria and the National Institute of Neurological Disorders and Stroke rt-PA Stroke Study Group (NINDS trial) criteria [14]. Follow-up ended at hospital discharge in $46 \%$ ( $n=16$ studies) of the studies, while in remaining studies, follow-up timeperiods ranged between 1 and 12 months. Among these studies, loss to follow-up varied between 0 and $26 \%$.

\section{Presentation}

Stroke Risk Factors

The prevalence of stroke risk factors varied across the different studies with substantial unexplained heterogeneity in the pooled estimates, which should be interpreted with caution. Hypertension was the most prevalent risk factor, based on data from 30 studies (61,738 patients), and was reported in $64 \%\left(95 \%\right.$ CI $\left.59-69, I^{2}=99 \%\right)$ of patients. Smoking was the second most common risk factor and was reported in 26 studies and 26,329 patients (25\%, 95\% CI $\left.19-32, I^{2}=99 \%\right)$. Based on results from 29 studies $(61,638$ patients), it was found that diabetes was nearly as common as smoking (23\%, 95\% CI 19-28, $\left.I^{2}=99 \%\right)$. Previous strokes were reported in 21 studies (55,391 patients), with an overall prevalence of $15 \%$ (95\% CI 9-23\%, $\left.I^{2}=100 \%\right)$. Atrial fibrillation was the least commonly identified risk factor (pooled prevalence $=9 \%$ [95\% CI 7-12, $I^{2}=98 \%$ ] based on data from 23 studies (58,897 patients; Table 1$)$. There was a high risk of publication bias based on funnel plot asymmetry across all risk factors (Eggers test, $p<0.01$ ). Estimates by country income group are presented in forest plots in the online supplementary Figures IV-VIII.

\section{Neurological Assessment at Presentation}

Information on loss of consciousness was reported in 9 studies (5,299 patients). Overall, 31\% (95\% CI 21-43, $\left.I^{2}=98 \%\right)$ of acute stroke patients had loss of consciousness at presentation to the hospital. In UMIC, loss of consciousness was reported among $44 \%$ (95\% CI 42-46) of patients, based on data from Brazil only, 25\% (95\% CI $11-42$ ) in LrMIC, and 38\% in LIC (95\% CI 21-43\%; online suppl. Fig. IX). Study authors reported results from the National Institutes of Health Stroke Scale only in 13 studies (35\%). These data were not pooled due to variations in presentation of results (online suppl. Table IV).

\section{Assessment of Swallow Function}

Swallow function was discussed only in 2 (5\%) studies. Among 148 stroke patients in Gambia, 65\% presented with swallow difficulties upon admission. In Vietnam, 
Table 1. Summary of pooled estimates of stroke risk factors.

\begin{tabular}{|c|c|c|c|c|}
\hline Risk factor & Overall (36 studies) & UMIC (14 studies) & LrMIC (18 studies) & LIC (4 studies) \\
\hline Hypertension & $\begin{array}{l}64 \%\left(95 \% \text { CI } 59-69, I^{2}=99 \%\right) \\
30 \text { studies, } 61,738 \text { participants }\end{array}$ & $\begin{array}{l}65 \%\left(95 \% \text { CI } 58-72, I^{2}=100 \%\right) \\
12 \text { studies, } 54,545 \text { participants }\end{array}$ & $\begin{array}{l}65 \%\left(95 \% \text { CI } 54-76, I^{2}=99 \%\right) \\
14 \text { studies, } 6,089 \text { participants }\end{array}$ & $\begin{array}{l}59 \%\left(95 \% \text { CI } 26-87, I^{2}=99 \%\right) \\
4 \text { studies, } 1,104 \text { participants }\end{array}$ \\
\hline Smoking & $\begin{array}{l}25 \%\left(95 \% \text { CI } 19-32, I^{2}=99 \%\right) \\
26 \text { studies, } 26,329 \text { participants }\end{array}$ & $\begin{array}{l}35 \%\left(95 \% \text { CI } 26-45, I^{2}=99 \%\right) \\
10 \text { studies, } 19,636 \text { participants }\end{array}$ & $\begin{array}{l}21 \%\left(95 \% \text { CI } 15-27, I^{2}=97 \%\right) \\
15 \text { studies, } 6,530 \text { participants }\end{array}$ & $\begin{array}{l}4 \%\left(95 \% \text { CI } 2-9, I^{2}=\text { NA }\right) \\
1 \text { study, } 163 \text { participants }\end{array}$ \\
\hline Diabetes & $\begin{array}{l}23 \%\left(95 \% \text { CI } 19-28, I^{2}=99 \%\right) \\
29 \text { studies, } 61,638 \text { participants }\end{array}$ & $\begin{array}{l}26 \%\left(95 \% \text { CI } 19-32, I^{2}=100 \%\right) \\
12 \text { studies, } 54,545 \text { participants }\end{array}$ & $\begin{array}{l}26 \%\left(95 \% \text { CI } 17-37, I^{2}=99 \%\right) \\
13 \text { studies, } 5,989 \text { participants }\end{array}$ & $\begin{array}{l}7 \%\left(95 \% \text { CI } 3-13, I^{2}=87 \%\right) \\
4 \text { studies, } 1,104 \text { participants }\end{array}$ \\
\hline Previous stroke & $\begin{array}{l}15 \%\left(95 \% \text { CI } 9-23, I^{2}=100 \%\right) \\
21 \text { studies, } 55,391 \text { participants }\end{array}$ & $\begin{array}{l}18 \%\left(95 \% \text { CI } 8-32, I^{2}=100 \%\right) \\
8 \text { studies, } 49,490 \text { participants }\end{array}$ & $\begin{array}{l}15 \%\left(95 \% \text { CI } 12-19, I^{2}=91 \%\right) \\
10 \text { studies, } 4,939 \text { participants }\end{array}$ & $\begin{array}{l}10 \%\left(95 \% \text { CI } 2-22, I^{2}=\text { NA }\right) \\
3 \text { studies, } 962 \text { participants }\end{array}$ \\
\hline Atrial fibrillation & $\begin{array}{l}9 \%\left(95 \% \text { CI } 7-12, I^{2}=98 \%\right) \\
23 \text { studies, 58,897 participants }\end{array}$ & $\begin{array}{l}10 \%\left(95 \% \text { CI } 7-14, I^{2}=99 \%\right) \\
11 \text { studies, } 53,894 \text { participants }\end{array}$ & $\begin{array}{l}9 \%\left(95 \% \text { CI } 5-14, I^{2}=96 \%\right) \\
9 \text { studies, } 4,062 \text { participants }\end{array}$ & $\begin{array}{l}6 \%\left(95 \% \text { CI } 4-8, I^{2}=\mathrm{NA}\right) \\
3 \text { studies, } 941 \text { participants }\end{array}$ \\
\hline
\end{tabular}

LIC, low-income country; LrMIC, lower-middle income country; UMIC, upper-middle income country; NA, not applicable.

Table 2. Summary of pooled estimates of evidence-based practices for evaluation and management of stroke

\begin{tabular}{|c|c|c|c|c|}
\hline & Overall (36 studies) & UMIC (14 studies) & LrMIC (18 studies) & LIC (4 studies) \\
\hline Head CT scan ${ }^{1}$ & $\begin{array}{l}90 \%\left(95 \% \text { CI } 79-97, I^{2}=99 \%\right) \\
6 \text { studies, } 7,595 \text { participants }\end{array}$ & $\begin{array}{l}99 \%\left(95 \% \text { CI } 98-99, I^{2}=\text { NA }\right) \\
2 \text { study, 5,626 participants }\end{array}$ & $\begin{array}{l}77 \%\left(95 \% \text { CI } 75-80, I^{2}=\text { NA }\right) \\
2 \text { studies, } 1,176 \text { participants }\end{array}$ & $\begin{array}{l}82 \%\left(95 \% \text { CI } 79-85, I^{2}=\text { NA }\right) \\
2 \text { studies, } 793 \text { participants }\end{array}$ \\
\hline MRI & $\begin{array}{l}32 \%\left(95 \% \text { CI } 9-48, I^{2}=100 \%\right) \\
8 \text { studies, } 8,335 \text { participants }\end{array}$ & $\begin{array}{l}39 \%\left(95 \% \text { CI } 2-96, I^{2}=100 \%\right) \\
4 \text { studies, } 6,431 \text { participants }\end{array}$ & $\begin{array}{l}26 \%\left(95 \% \text { CI } 8-48, I^{2}=99 \%\right) \\
4 \text { studies, } 1,904 \text { participants }\end{array}$ & 0 studies \\
\hline rt-PA use ${ }^{2}$ & $\begin{array}{l}3 \%\left(95 \% \text { CI } 2-4, I^{2}=97 \%\right) \\
10 \text { studies, } 44,4928 \text { participants }\end{array}$ & $\begin{array}{l}1 \%\left(95 \% \text { CI } 1-2, I^{2}=95 \%\right) \\
8 \text { studies, } 44,216 \text { participants }\end{array}$ & $\begin{array}{l}24 \%\left(95 \% \text { CI } 19-29, I^{2}=\text { NA }\right) \\
2 \text { studies, } 276 \text { participants }\end{array}$ & 0 studies \\
\hline Antiplatelet therapy & $\begin{array}{l}78 \%\left(95 \% \text { CI } 66-88, I^{2}=100 \%\right) \\
8 \text { studies, } 44,044 \text { participants }\end{array}$ & $\begin{array}{l}79 \%\left(95 \% \text { CI } 66-90, I^{2}=\mathrm{NA}\right) \\
6 \text { study, } 43,148 \text { participants }\end{array}$ & $\begin{array}{l}49 \%\left(95 \% \text { CI } 46-53, I^{2}=\mathrm{NA}\right) \\
1 \text { study, } 754 \text { participants }\end{array}$ & $\begin{array}{l}94 \%\left(95 \% \text { CI } 88-97, I^{2}=\text { NA }\right) \\
1 \text { study, } 142 \text { participants }\end{array}$ \\
\hline Admitred to stroke unit & 3 studies, data not pooled & $\begin{array}{l}\text { Thailand: } 25 \% \text {; } \\
\text { Brazil: } 12 \% \text {; } \\
\text { Argentina: } 6 \%\end{array}$ & 0 studies & 0 studies \\
\hline Admitted to ICU & $\begin{array}{l}18 \%\left(95 \% \text { CI } 12-24, I^{2}=98 \%\right) \\
5 \text { studies, } 35,069 \text { participants }\end{array}$ & $\begin{array}{l}14 \%\left(95 \% \text { CI } 7-21, I^{2}=\mathrm{NA}\right) \\
3 \text { studies, } 33,990 \text { participants }\end{array}$ & $\begin{array}{l}26 \%\left(95 \% \text { CI } 23-29, I^{2}=\mathrm{NA}\right) \\
2 \text { studies, } 1,079 \text { participants }\end{array}$ & 0 studies \\
\hline Early hospital mobilization & 0 studies & 0 studies & 0 studies & 0 studies \\
\hline Early rehabilitation & 2 studies, data not pooled & $\begin{array}{l}\text { Brazil: } 77 \% \text { physical therapy; } \\
32 \% \text { speech therapy }\end{array}$ & $\begin{array}{l}\text { Vietnam: } 6 \% \text { occupational therapy; } \\
2 \% \text { physical therapy; } \\
<1 \% \text { speech therapy }\end{array}$ & 0 studies \\
\hline
\end{tabular}

${ }^{1}$ Reported among studies that did a CT scan only without an MRI or other more advanced neuroimaging.

${ }^{2}$ Excludes studies that limited the inclusion criteria to rt-PA treatment only.

LIC, low-income country; LrMIC, lower-middle income country; UMIC, upper-middle income country; NA, not applicable; ICU, intensive care unit.

$16 \%$ of patients presenting with ischemic stroke and $6 \%$ of patients presenting with hemorrhagic stroke had dysphagia at presentation.

Time from Stroke Onset to Hospital Admission

The inclusion criteria were restricted to patients who received rt-PA in 5 studies, authors report the time from stoke onset to hospital admission in 4 of these studies. The mean (SD) number of hours ranged from 1 (0.2) h in Serbia to $3.5 \mathrm{~h}$ (SD not reported) in Morocco. In the remaining studies where the inclusion criteria was not restricted to patients who received rt-PA, the mean time ranged from 3 (1) $\mathrm{h}$ in India to 47 (19) $\mathrm{h}$ in Cameroon (online suppl. Table V).

\section{Evaluation and Management of Acute Stroke}

Pooled estimates for the evaluation and management of stroke are summarized in Table 2 and in online supplementary Figures X-XIV. 
Imaging

Twenty-six studies provided information on the proportion of patients who received a head CT scan during their admission. In 11 of these studies, receiving a CT scan was part of the inclusion criteria and in another 8 studies, patients received a CT scan, MRI, or both. Based on the remaining 6 studies, CT scans were used among $90 \%$ of patients ( $95 \%$ CI $79-97, I^{2}=99 \%$ ).

Eight studies provided information on the use of CT scans and MRIs. MRI use was reported among 32\% (95\% CI 9-61, $\left.I^{2}=100 \%\right)$ of patients. Data on MRI use were based on countries from UMIC and LrMIC only because none of the LIC reported MRI use.

Thrombolysis and Antiplatelet Therapy

We identified 14 studies that provided evidence on rtPA use, 4 of which limited the inclusion criteria to patients receiving treatment only, and thus these studies were excluded from the pooled analysis. Overall, 3\% (95\% CI 2-4, $I^{2}=97 \%, 10$ studies) of patients were treated with rt-PA. None of the studies in LIC provided information on using rt-PA. Seventy-eight percent $\left(95 \%\right.$ CI 66-88, $I^{2}=$ $100 \%, 8$ studies) of patients were treated with acute antiplatelet therapy.

There was no risk of publication bias based on funnel plot asymmetry for rt-PA use (Eggers test, $p=0.553$ ).

Stroke and Intensive Care Unit Admissions

Admission to organized stroke units was discussed in 3 studies, with $25 \%$ of patients admitted in Thailand, $12 \%$ in Brazil, and 6\% in Argentina. A fourth study conducted in Vietnam, included only stroke patients that were admitted to the stroke unit. Authors from 5 studies, reported admitting patients to an intensive care unit with a pooled prevalence of $18 \%$ (95\% CI $\left.12-24 \%, I^{2}=98 \%\right)$.

Rehabilitation and Early In-Hospital Mobilization

None of the studies provided information on early inhospital mobilization, while in-hospital rehabilitation was discussed in 2 studies. In Vietnam, $6 \%$ of stroke patients received occupational therapy, $2 \%$ received physical therapy, and less than $1 \%$ received speech therapy. In Brazil, $77 \%$ of stroke patients received physical therapy and $32 \%$ received speech therapy.

\section{Outcomes}

In-hospital mortality ranged from $1 \%(95 \%$ CI 1-2) in Mexico to $45 \%$ (95\% CI 35-56) in Nigeria (27 studies, 24,857 patients; Fig. 1). Overall, in-hospital mortality was 14\% (95\% CI 10-19, $\left.I^{2}=99 \%\right)$. In UMIC, in-hospital mor- tality was $9 \%$ (95\% CI 4-16, $I^{2}=99 \%, 8$ studies). Mortality was higher in LrMIC (15\%, 95\% CI 9-22, $I^{2}=98 \%, 15$ studies) and highest in LIC (24\%, 95\% CI 13-38, $I^{2}=95 \%$, 4 studies).

Pneumonia ranged from 6\% (95\% CI 5-8\%) in Thailand to $45 \%$ (95\% CI 38-53\%) in Tanzania (14 studies, 19,136 patients; Fig. 2). Overall, pneumonia was reported among $17 \%$ (95\% CI 14-20) of patients. Rates of pneumonia were lower in UMIC (13\%, 95\% CI 10-17, $\left.I^{2=} 97 \%\right)$ compared to LrMIC $\left(20 \%, 95 \%\right.$ CI $\left.13-28, I^{2}=96 \%\right)$ and LIC (19\%, 95\% CI 15-24).

There was a high risk of publication bias based on funnel plot asymmetry for in-hospital mortality and pneumonia (Eggers test for both, $p<0.05$ ).

\section{Discussion}

Results from our systematic review show that the use of evidence-based practices is suboptimal in LMIC. This was true for practices that have been identified for advanced settings (e.g., MRI and rt-PA) as well as practices that should be feasible in settings with minimal resources (e.g., antiplatelet therapy, and neurological assessments). The review also demonstrates that MRI and rt-PA were absent in LIC. Furthermore, evidencebased practices were most commonly used in UMIC and least commonly used in LIC. These inferences should be interpreted with caution because the number of studies within each country income group is small. Results from INTERSTROKE, an international observational cohort study of 12,342 stroke patients from 108 hospitals in 25 high-, middle-, and LIC, supports results from this review and shows a similar gradient of higher use of evidence-based practices for stroke in HIC and least in LIC [15].

Though the prevalence of stroke risk factors was lower in LIC compared to UMIC and LrMIC, patients in LIC had the highest in-hospital mortality. This apparent paradox is consistent with reports from previous, community-based data [16], and may represent delays in presentation for seeking acute stroke care, differences in health system response for acute stroke management, or both.

As expected, our review shows that use of these practices in LMIC is lower than it is in studies conducted in HIC. For example, our review revealed pooled estimates of $90 \%$ for head CT scans and $78 \%$ for antiplatelet therapy. The use of these practices vary in HIC; results from Get With the Guidelines (GWG) indicate that use of 
Fig. 1. In-hospital mortality based on the country income group.

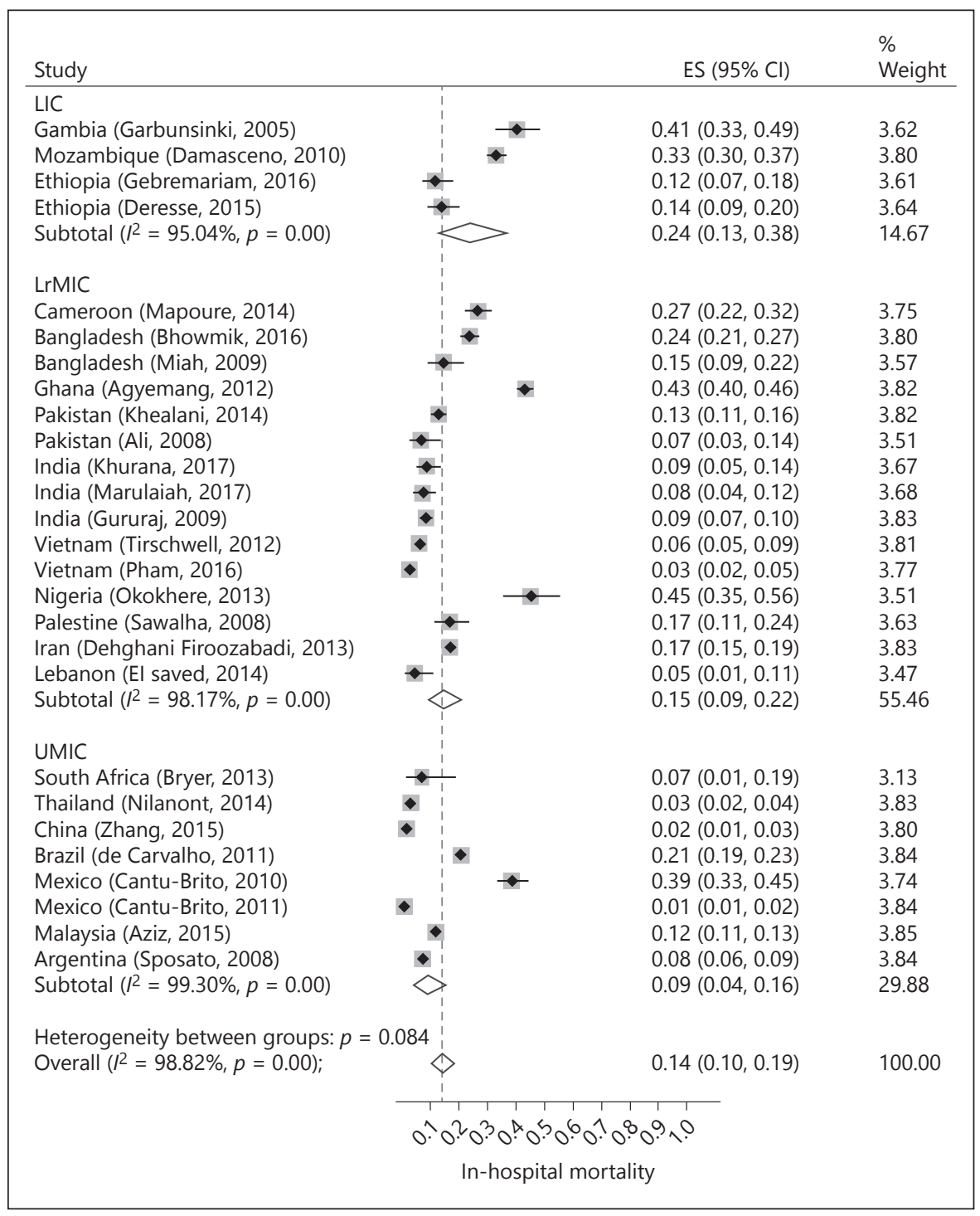

head CT scans is close to $100 \%$ [17] and $92 \%$ receive early antiplatelet therapy [18]. However, rates of using these evidence-based practices are likely to be lower and closer to results from our review in hospitals that do not participate in Get With the Guidelines. Further, rt-PA use is close to $30 \%$ in HIC [18] but was reported only in $3 \%$ of patients in our review. In most studies, authors reported only using acute antiplatelet therapy indicating that rtPA use in many LMIC is even lower than the pooled estimate and in many regions is closer to $0 \%$. The high cost of altepase is commonly reported as a barrier to thrombolysis in stroke patients and is often not covered by insurance [19].

Organized stroke units, which are the single most effective organizational intervention to improving stroke outcomes [4], were reported only in 4 of the studies, and less than $50 \%$ of patients were admitted into these stroke units. It is important to keep in mind that these practices remain suboptimal even in HIC hospitals, especially in rural areas. For example, a 2013 study conducted in the United States revealed that the number of hospitals that have organized stroke unites varied widely across the states, ranging from $4 \%$ in Wyoming to $100 \%$ in Delaware, with an overall median of $25 \%$ [20]. This confirms the continuous need to improve and build on our knowledge of implementing evidence-based practices for stroke care across all country income groups. Success stories of implementing practices such as organized stroke units from LMIC [21] could be used to increase the use of these organizational interventions not only in 
Fig. 2. Percent of patients with pneumonia based on the country income group.

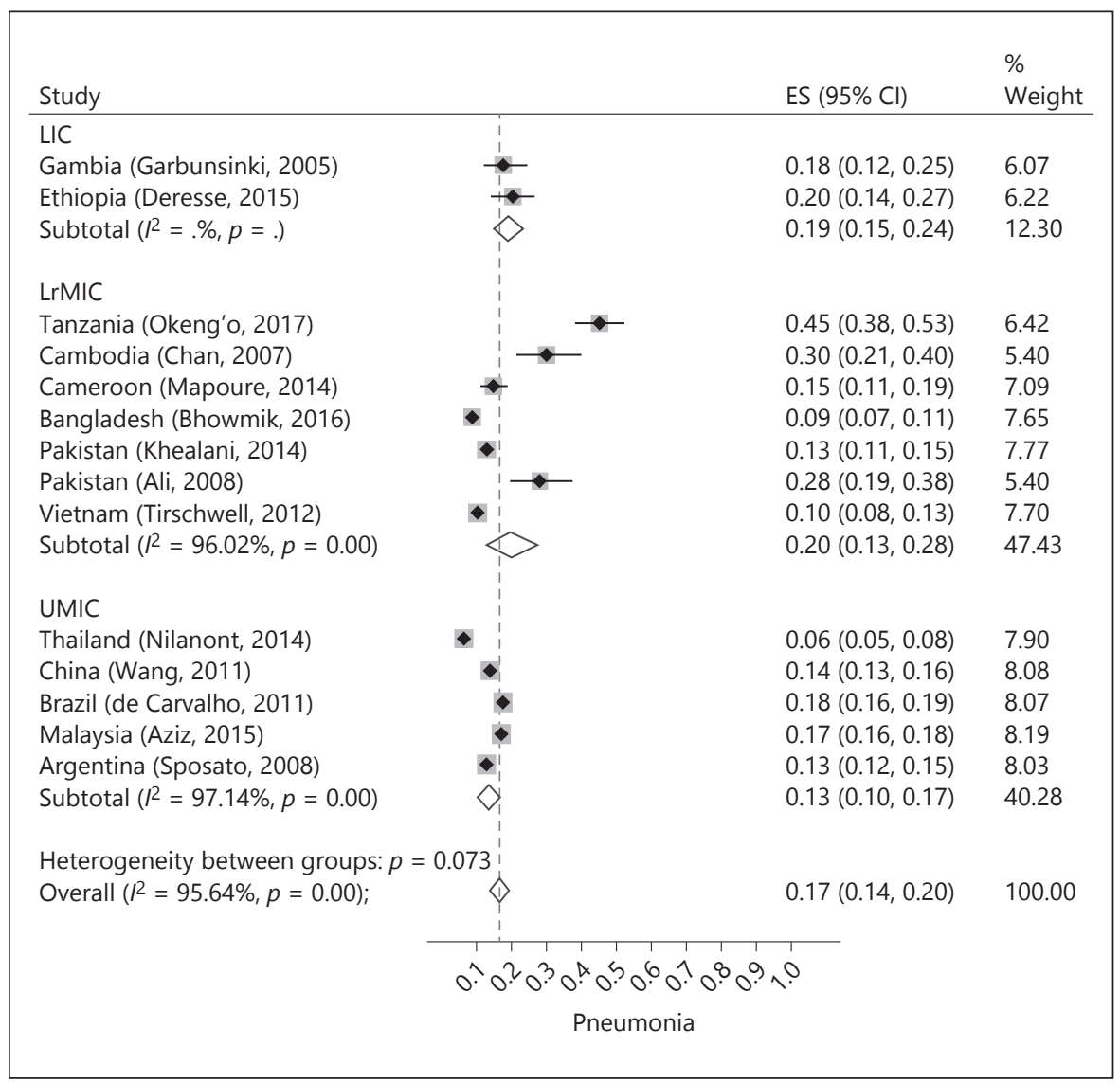

other LMIC but also in HIC that do not currently have them.

We referred to the Global Stroke Services Action Plan developed by the World Stroke Organization to guide our question on evaluating and managing stroke. This plan was conceived as a tool to identify key evidence-based practices across a continuum of health models in which these practices may be realistically carried out [6]. Targeting feasible best practices is an important goal in low resource settings that do not have the capacity to develop stroke units.

In this review, we were able to pool estimates from 36 studies describing stroke presentation patterns, evaluation and management, and outcomes in LMIC. Our review compliments a 2017 systematic review that highlights existing stroke services that are evidence-based and implementable in LMICs. Examples of these services include acute ischemic stroke protocols, low-cost tele imaging, and intravenous thrombolysis guided by a telemedicine consultation system. The authors conclude that further research on effectiveness and suitability of these strategies is needed [21]. A 2015 systematic review of 101 studies of community-based stroke survival in LMIC showed that annual stroke survival rates were the highest in Latin American and Caribbean countries (21.2 per 1,000, 95\% CI 13.7-30.29) and lowest in sub-Saharan Africa countries (3.5 per 1,000, 95\% CI 1.9-5.7) [11]. However, information on acute stroke management was not reported. Yan et al. [22] also reviewed the literature on stroke in LMIC, describing risk factors, current trends in primary prevention, management of acute stroke, secondary prevention, and rehabilitation. Unlike Yan's review, which is a narrative synthesis of the literature, we were able to provide pooled estimates to quantify the data on acute stroke care in LMIC settings. Another strength is the diversity of included studies in terms of regions and country income groups. We were able to show a trend for hospital mortality, with pneumonia being highest in LIC and lowest in UMIC.

Our study has limitations. First, summary results should be interpreted with caution, given the large observed heterogeneity in the pooled estimates and study limitations, including high risk of publication bias in some domains. Heterogeneity was not explained by the 
study setting, but variations in study design and inclusion criteria likely contribute. For example, $36 \%$ of the studies restricted inclusion to ischemic strokes only therefore skewing results toward practices used in managing ischemic strokes rather than any stroke type. Heterogeneity may also be explained by variations in healthcare systems, which are observed even within the same region and across countries with similar incomes. Reporting methods were variable in the studies, and in some cases, limited our ability to pool estimates. Second, we restricted the time of our search and thus did not capture earlier studies before 2005. However, it is uncertain how relevant prior studies may be for current and future health system assessment and planning. Third, our review was limited by the relatively low number of reports for such a common condition, especially from LIC. This demonstrates the need for facility-based surveillance to evaluate and improve health system performance for acute stroke care, such as the WHO STEPwise stroke surveillance tool [23]. Fourth, sites that do this kind of research are likely limited to large teaching hospitals in each country, mostly in urban locations. Therefore, estimates from our review may not reflect the use of evidence-based practices in smaller, nonteaching hospitals nor in hospitals located in rural and secluded regions of LMICs. The "true use" of these evidence-based practices is likely to be even lower than what we found in this review, suggesting an even great- er need for implementing organized stroke units and make thrombolysis available and affordable. These strategies improve stroke outcomes and appear to be feasible in LMIC [21].

In conclusion, our results provide a detailed overview of how stroke patients are managed in LMIC where stroke mortality and other complications are the highest. Our review indicates that even components that require minimal resources, such as assessments to prevent complications (e.g., neurological and swallow function assessment), acute antiplatelet therapy, and early mobilization, may not be available. Strategies to improve stroke care that have been successfully implemented in HIC may be useful for adaptation and implementation to LMIC settings to improve stroke care and outcomes.

\section{Disclosure Statement}

M.D.H. receives funding from the World Heart Federation to serve as its senior program advisor for the Emerging Leaders program, which is sponsored by Boehringer Ingelheim and Novartis with previous support from BUPA and AstraZeneca.

\section{Funding Source}

Funding was received from the NINDS grant number U10NS086608.

\section{References}

1 Feigin VL, Krishnamurthi RV, Parmar P, Norrving B, Mensah GA, Bennett DA, et al: Update on the global burden of ischemic and hemorrhagic stroke in 1990-2013: the GBD 2013 Study. Neuroepidemiology 2015;45:161-176.

2 Roth GA, Johnson C, Abajobir A, Abd-Allah F, Abera SF, Abyu G, et al: Global, regional, and national burden of cardiovascular diseases for 10 causes, 1990 to 2015. J Am Coll Cardiol 2017;70:1-25.

3 Schwamm LH, Pancioli A, Acker JE 3rd, Goldstein LB, Zorowitz RD, Shephard TJ, et al: Recommendations for the establishment of stroke systems of care: recommendations from the American Stroke Association's Task Force on the Development of Stroke Systems. Stroke 2005;36:690-703.

4 Langhorne P, de Villiers L, Pandian JD: Applicability of stroke-unit care to low-income and middle-income countries. Lancet Neurol 2012;11:341-348.

5 World Health Organization: from MDGs, millennium development goals to SDGs, sus- tainable development goals. World Health Organization 2015:204. http://www.who.int/ gho/publications/mdgs-sdgs/en/(accessed September 15, 2017).

6 Lindsay P, Furie KL, Davis SM, Donnan GA, Norrving B: World Stroke Organization global stroke services guidelines and action plan. Int J Stroke 2014:9(suppl A100): 4-13.

7 Moher D, Liberati A, Tetzlaff J, Altman DG; PRISMA Group: Preferred reporting items for systematic reviews and meta-analyses: the PRISMA statement. J Clin Epidemiol 2009; 62:1006-1012.

8 LMIC Filters: http://epoc.cochrane.org/lmicfilters. Cochrane Effective Practice and Organization of Care (EPOC) Group, 2013 (accessed 2017 May 23).

9 World Bank Country and Lending Groups: https://datahelpdesk.worldbank.org/knowledgebase/articles/906519-world-bank-country-and-lending-groups. World Bank, 2017 (accessed 2017 May 23).
10 Search Filters for Various Databases: Ovid Medline. http://libguides.sph.uth.tmc.edu/ search_filters/ovid_medline_filters. UTHealth School of Public Health, 2017 (accessed 2017 May 23).

11 Ezejimofor MC, Chen YF, Kandala NB, Ezejimofor BC, Ezeabasili AC, Stranges S, Uthman OA: Stroke survivors in low- and middle-income countries: a meta-analysis of prevalence and secular trends. J Neurol Sci 2016;364:68-76.

12 Egger M, Davey Smith G, Schneider M, Minder C: Bias in meta-analysis detected by a simple, graphical test. BMJ 1997;315:629-634.

13 Callender T, Woodward M, Roth G, Farzadfar F, Lemarie JC, Gicquel S, et al: Heart failure care in low- and middle-income countries: a systematic review and meta-analysis. PLoS Med 2014;11:e1001699.

14 National Institute of Neurological Disorders and Stroke rt-PA Stroke Study Group: Tissue plasminogen activator for acute ischemic stroke. N Engl J Med 1995;333:1581-1587. 
15 Langhorne P, O'Donnell MJ, Chin SL, Zhang $\mathrm{H}$, Xavier D, Avezum A, et al: Practice patterns and outcomes after stroke across countries at different economic levels (INTERSTROKE): an international observational study. Lancet 2018;391:2019-2027.

16 Yusuf S, Rangarajan S, Teo K, Islam S, Li W, Liu L, et al: Cardiovascular risk and events in 17 low-, middle-, and high-income countries. N Engl J Med 2014;371:818-827.

17 Kelly AG, Hellkamp AS, Olson D, Smith EE, Schwamm LH: Predictors of rapid brain imaging in acute stroke. Stroke 2012;43:12791284.
18 Reeves MJ, Grau-Sepulveda MV, Fonarow GC, Olson DM, Smith EE, Schwamm LH: Are quality improvements in the get with the guidelines: stroke program related to better care or better data documentation? Circ Cardiovasc Qual Outcomes 2011;4: 503-511.

19 Abd-Allah F, Moustafa RR: Burden of stroke in Egypt: current status and opportunities. Int J Stroke2014;9:1105-1108.

20 Uchino K, Man S, Schold JD, Katzan IL: Stroke legislation impacts distribution of certified stroke centers in the united states. Stroke 2015;46:1903-1908.
21 Pandian JD, William AG, Kate MP, Norrving B, Mensah GA, Davis S, et al: Strategies to improve stroke care services in low- and middleincome countries: a systematic review. Neuroepidemiology 2017;49:45-61.

22 Yan LL, Li C, Chen J, Miranda JJ, Luo R, Bettger J, et al: Prevention, management, and rehabilitation of stroke in low- and middle-income countries. eNeurologicalSci 2016;2:21-30.

23 The WHO STEPwise approach to stroke surveillance. 0 -version 1.2. World Health Organization. http://www.who.int/ncd_surveillance/en/steps_stroke_manual_v1.2.pdf. (accessed August 3, 2017). 